\title{
Energetic Materials Performance Enhancement Through Predictive Programming the Spatial Structure and Physics-Chemical Properties of the Functionalized Carbon-Based Nano-Sized Additives
} [Invited Talk]

\author{
Alexander Lukin \\ Western-Caucasus Research Center \\ Tuapse, 352808, Russian Federation \\ E-Mail: lukin@wcrc.ru
}

\begin{abstract}
A new generation of nano-technologies is expanding solid propulsion capabilities and increasing their relevance for versatile and manoeuvrable micro-satellites with safe high-performance propulsion. We propose the innovative concept, connected with application of new synergistic effect of the energetic materials performance enhancement and reaction zones programming for the next generation small satellite multimode solid propulsion system. The main idea of suggested concept is manipulating by the self-organized wave patterns excitation phenomenon, by the properties of the energetic materials reaction zones and by localization of the energy release areas. This synergistic effect can be provided through application of the functionalized carbon-based nanostructured metamaterials as a nano-additives along with simultaneous manipulating by their properties through the electrostatic field. Mentioned effect will be controlled through predictive programming both by the spatial structure and physicschemical properties of the functionalized carbon-based nano-additives and through the electromagnetic control of the self-organized wave pattern excitation and micro- and nano- scale oscillatory networks in the energetic material reaction zones. Suggested new concept makes it possible to increase the energetic material regression rate and increase the thrust of the solid propulsion system with minimal additional energy consumption.
\end{abstract}

Keywords: energetic materials; solid propulsion systems; extreme thrust control; reaction zones; functionalized carbon-based nanostructured metamaterials; nano-sized additives; carbon atomic wires, sp1-hybridized bonds; ionassisted pulsed-plasma deposition; self-organizing of the nanostructures; universal phenomena of nano-cymatics; electrostatic field; synergistic effect

\section{Introduction}

There is growing demand for in-space propulsion systems with extreme and precise thrust control that enable small satellites to achieve attitude and orbit control, orbital transfers, and end-of-life deorbiting. Next generation of the smart solid propulsion systems (SPS) while providing propulsion capability for small upper stages, also can be used for orbital maneuvers, attitude control, station keeping, constellation management, decommission and deorbit with very precise and predictable delivered impulse. SPS continue to be a reliable way to provide thrust and are used in almost every Earth-to-orbit launch capability. 
A new generation of nano-technologies is expanding solid propulsion capabilities and increasing their relevance for versatile and maneuverable micro-satellites with safe high-performance propulsion.

The micro- and nano- scale oscillatory networks arising in the solid propellant reaction zones has a significant influence on physical and chemical processes and on controllability of ignition and combustion processes.

The analysis of experimental data shows that the macro-scale phenomena at the energetic materials (EM) combustion are result of self-organizing and self-synchronization of the micro- and nano- scale oscillatory networks in the EM reaction zones. Programming by self-organized wave patterns excitation is one of the keys to access to the properties of the EM reaction zones. Behind excitation of the micro- and nano- scale oscillatory networks and wave patterns exists the universal Cytmatics phenomenon, [1].

A number of the experimental researches shows that functionalized carbon-based nanostructures provide a significant effect on thermal decomposition, ignition, combustion and reactivity properties of the EM and solid propellants, [2-5]. In particular, inserting of the detonation nano-diamonds into the solid propellant composition changes the thermal decomposition process and leads to increase of the burning rate, $[6,7]$.

It has been shown that use of functionalized carbon-based nanostructures in the EM would greatly improve their combustion performances, thermal stability and sensitivity, [3-5]. Extensive investigations were and are conducted regarding the design, synthesis, characterization and application of functionalized carbon-based nanostructures in the EM. Use of various functionalized carbon-based nanostructured metamaterials as a nano-additives in the EM is extremely promising, opens possibilities solve numerous problems in this field and having extremely capabilities in the nearest future.

In this connection, manipulating by the special structure and physics-chemical properties of the carbonbased nanostructured additives opens the new possibilities for enhancement of the solid propellants and EM performances.

We suggest the innovative concept, connected with application of new synergistic effect of the EM performance enhancement and reaction zones programming for the next generation small satellite multimode SPS. In such kind of multimode SPS the one propulsion system with one propellant can operates in the chemical mode with high thrust, or can operate in an electric mode, in which a very fast electric pulse ablates the surface off of the material, and provides very high performance, such as the ablative pulsed plasma thruster.

The main idea of suggested concept is manipulating by the self-organized wave patterns excitation phenomenon, by the properties of the EM reaction zones and by localization of the energy release areas.

This synergistic effect can be provided through application of the functionalized carbon-based 
nanostructured metamaterials as a nano-additives along with simultaneous manipulating by their properties through the electrostatic field. Mentioned effect will be controlled through predictive programming both by the spatial structure and physics-chemical properties of the functionalized carbon-based nanostructured metamaterial and through the electromagnetic control of the self-organized wave pattern excitation and micro- and nano- scale oscillatory networks in the EM reaction zones.

The properties of the carbon-based nanostructured metamaterials essentially depend on their spatial structure. Differently hybridized carbon atoms are capable to form diamond, graphite, linear-chain grids and many other specific allotropes. Each carbon allotrope has notably different electronic and mechanical properties. Carbon allotropes can function either as a conductor, in sp-1 chains, carbynes, and sp-2 planar structures, graphene and graphite, or as a wide-gap insulator in sp-3 tethrahedral coordination - e.g., diamond and alkanes. The first two have the potential to form bonds that are electrically conducting, while sp-3 has insulating properties. For instance, carbon nanotube demonstrates remarkable electrical conductivity and anisotropic electromagnetic characteristics, [8] that represent a great potential for different practical applications.

\section{Predictive Functionalization of the Carbon-Based Nanostructured Metamaterials}

Let's consider the new technological possibilities for functionalization of the carbon-based nanostructured metamaterials for application as nano-additives in the EM and solid propellants.

Sp-hybridized carbon atoms chains or carbon atomic wires (CAWs), called also as carbyne, represent the ultimate and lightweight one-dimensional (1D) system with exceptional physics-chemical properties. Carbyne properties can be manipulated through the chain length, doping by nano-clusters, and by type of the chain termination. However, the growth of the macroscopic crystals of the carbyne (CAWs) is inhibited by the instability and high reactivity of this allotropic form of carbon. For practical use of carbine-like nanostructured metamaterials, the ability to ensure high stability of this nanomaterial is of key importance. Nanostructures stability depends from the linear carbon chains length. Free CAWs of any length must be terminated by molecular complexes to ensure their stability. Number of experiments show the sp1-bonds and $\mathrm{sp-hybridized} \mathrm{carbon} \mathrm{nanostructures} \mathrm{are} \mathrm{formed} \mathrm{only} \mathrm{in} \mathrm{a} \mathrm{narrow} \mathrm{range} \mathrm{of} \mathrm{optimal} \mathrm{grows} \mathrm{parameters.}$

In 2016, a fundamentally new strategy was proposed and demonstrated to ensure the stability of the structure of the extremely long sp1 hybridized carbon chains containing more than 6000 carbon atoms through growing within the long nano-matrices formed by the double-walled carbon nanotubes, [9]. The thin double-walled carbon nanotubes surround the 1D carbyne molecule and protect it from inevitable 
disintegration. This result showed the fundamental possibility of using the control of the nano-matrix structure for programming the stability of the carbon chains.

The strategy, connected with the growth of the sp-hybridized carbon nanostructures in the composition of multi-cavity nano-matrices seems to be the most promising way for the creation of the advanced carbonbased nanostructured metamaterials.

Relatively recently, a method was found to compensate for the above disadvantages by using the technology of ion-plasma growing of stable carbine-like nanostructures in the composition of multi-cavity nano- matrixes, [10]. Such nano-sized carbon structures obtained the name as two-dimensionally ordered linear-chain carbon, which is a two-dimensionally packed hexagonal array of carbon chains held by van der Waals forces at a distance of about 5 angstroms.

As a functionalized carbon-based nano-additives for EM and solid propellants we propose to consider the two-dimensionally ordered linear chain carbon nano-matrix. Mentioned nano-matrix also can be represented as a nano-matrix of the CAWs, containing sp1-hybridized bonds or carbyne-like nano-matrix.

The ordered array of the one-dimensional CAWs are oriented perpendicular to the substrate surface and are densely packed parallel to one another in hexagonal structures with the interchain spacing approximately being between $4.8-5.03 \AA$. Therefore, the CAWs very weakly interacting with each other (due to Van der Waals interaction). Van der Waals is a weak force that allows neutral molecules to attract one another through randomly fluctuating dipoles, depending on distance.

The vibration of a carbon chains inside the multi-cavity nano-matrix occurs due to the van der Waals interactions between them.

Such kind of nano-matrix represent a multi-cavity nanostructure, containing a vacant functional nanocavities, available for assembling by atom clusters of other chemical elements, and in particular, the specific catalytic agents. The two-dimensionally ordered linear chain carbon nano-matrix could serve as an efficient basis for designing and the growth of the new carbon-based nanostructured metamaterials with unique electrophysical, optical, structural, topographic, biological and chemical properties. The clusterassembling of the spatial structure of two-dimensionally ordered linear-chain carbon nano-matrix with various specific catalytic agents and chemical elements fundamentally change the properties of the source nano-matrix. The spatial structure of such kind of nano-matrix can also self-adjusts to the structure of the embedded atom clusters. Geometric characteristics of the two-dimensionally ordered linear-chain carbon nano-matrix is presented on Figure 1. 


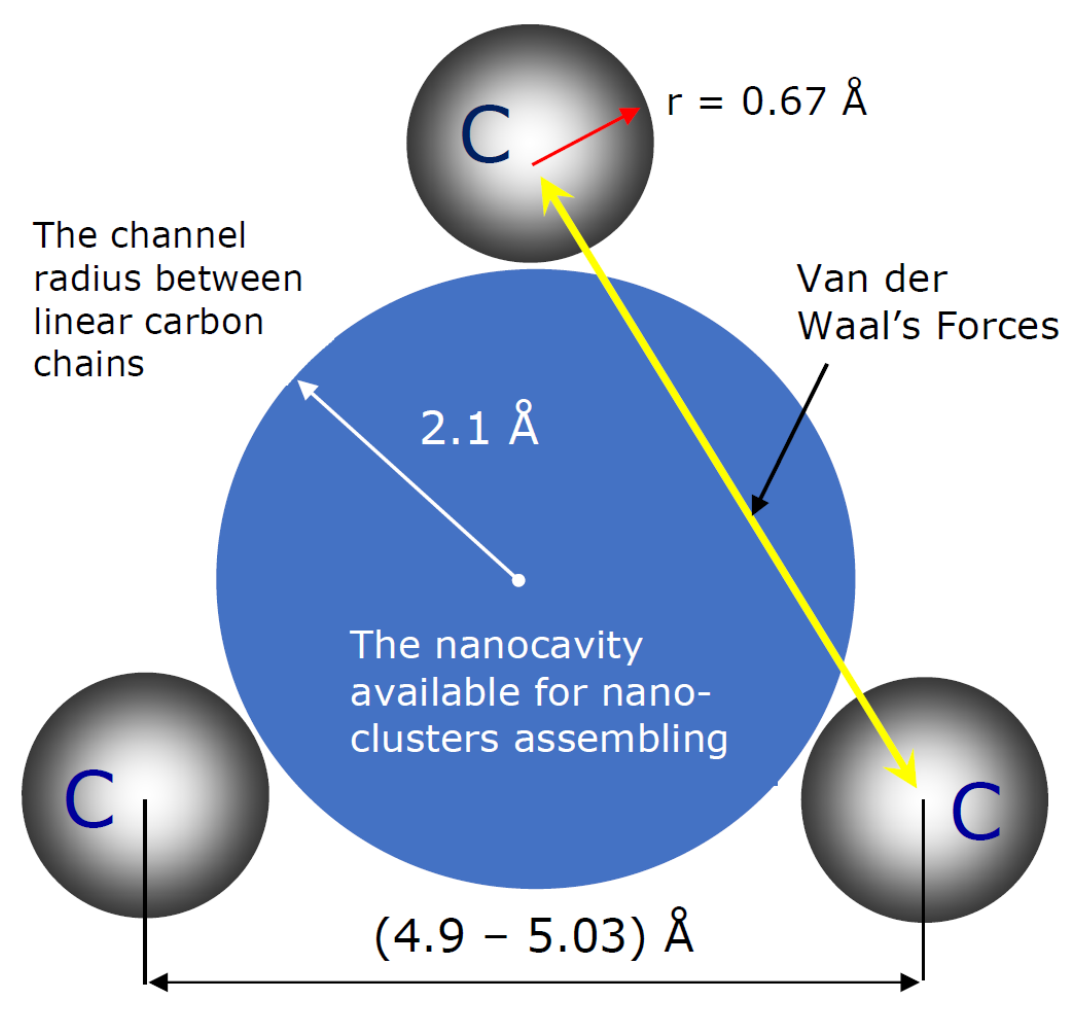

The distance between carbon chains

Figure 1. Geometric characteristics of the two-dimensionally ordered linear-chain carbon nano-matrix cell with the vacant functional nanocavity, available for the atomic nano-clusters assembling.

For instance, assembling the two-dimensionally ordered linear-chain carbon nano-matrix with calcium clusters, which suck up hydrogen molecules, creates a high-density, reversible hydrogen storage device.

This nano-matrix also can be considered as both acoustic and electromagnetic sensitive nanostructured metamaterial. The metamaterials derive their unique properties not from the properties of the base materials, but from their newly designed structures. The metamaterials are usually arranged as a repeated pattern, at a scale that are smaller than the wavelengths of the phenomena they influence. Metamaterials present exceptional properties, dominated by their geometrical structure. The precise shape, geometry, size, orientation and arrangement of the metamaterials gives them a number of smart properties capable of manipulating both acoustic and electromagnetic waves to achieve benefits that go beyond what is possible at using of the conventional nanomaterials. 


\section{Pulse-Plasma Experimental Set-Up}

The experimental set-up for ion-assisted pulse-plasma deposition of the two-dimensionally ordered linearchain carbon nano-matrix with capability of cluster-assembling by various specific catalytic agents and chemical elements are presented at the Figure 2.

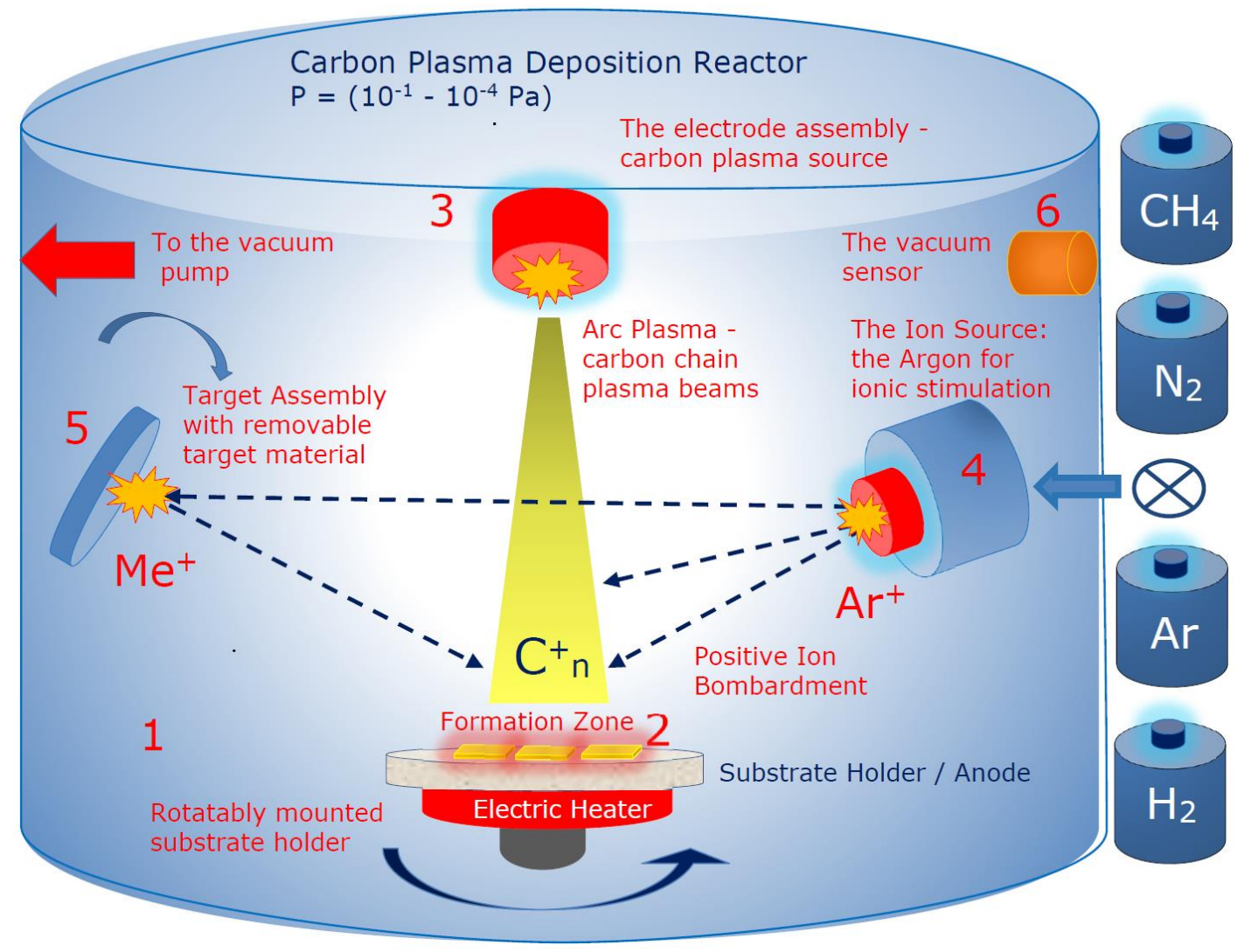

Figure 2. Schematic representation of the carbon pulse-plasma deposition reactor for growing of the carbyne-based nanostructures: 1 - vacuum chamber; 2 - substrate; 3 - pulse-plasma carbon generator (graphite cylindrical cathode of main discharge); 4 - the ion source for ionic stimulation; 5 - target assembly with removable target material; 6 - vacuum sensor.

The ion beam irradiation of the substrate surface forms bends in the attached carbon chains which stabilize the growing chain ensemble. The ion and plasma beams intersect at the substrate surface. The evaporation of the carbon plasma sheaf from the graphite cathode of main discharge 3 is caused by local heating of the graphite surface by electron bombardment to $T=3000^{\circ} \mathrm{C}$. The chains of carbon atoms, $C_{n}$ (where $n=1,2,3$, ...), formed in the plasma sheaf are directed by electrodes to impinge upon the surface of the substrate where the polycondensation of the carbon chains takes place. The schematic representation of the pulse- 
plasma carbon generator that installed in the reactor of the experimental set-up (Figure 2) are presented at the Figure 3.

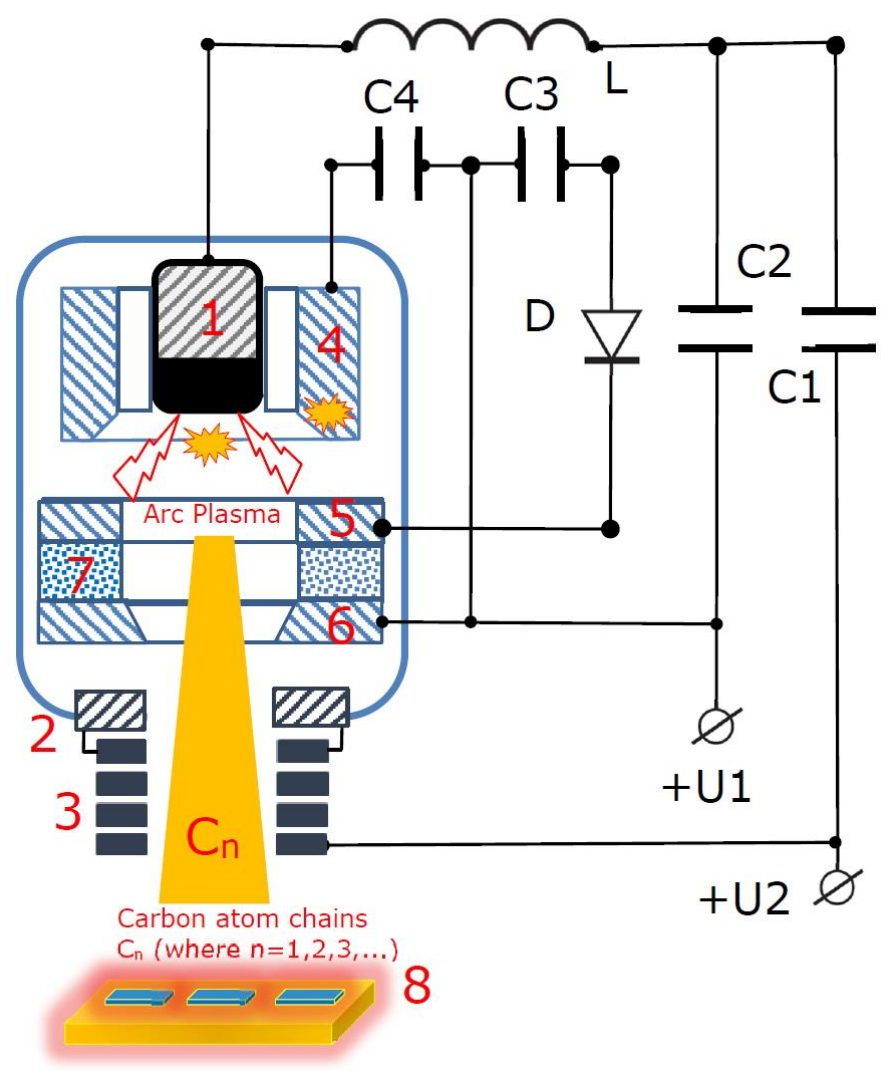

Figure 3. Schematic representation of the pulse-plasma carbon generator that installed in the reactor of the experimental set-up (Fig. 1): 1 - a cylindrical cathode of main discharge (evaporated material, high purity graphite); 2 - anode of the main discharge; 3 - a solenoid final focusing system with plasma neutralization; 4 - second anode of auxiliary discharge; 5 - ignition electrode; 6 - cathode of auxiliary discharge; 7 dielectric insulator; 8 - substrate holder.

An arc discharge is ignited between the cathode of main discharge 1 and anode of main discharge 2 (which are preferably separated by a voltage of about $200 \mathrm{~V}$ ) by means of auxiliary discharge between the cathode of auxiliary discharge 6 and the cathode of main discharge 1 and the anode of auxiliary discharge 4 surrounding the cathode of main discharge 1. The auxiliary discharge is ignited by means of ignition electrode 5. The design of the cathode (item 1 in Fig. 3) depends from the purposes of deposition and can be manufactured as a composite structure, containing cylindrical rods from different materials, for instance, silver, tungsten, gold etc. The example of design of a cylindrical cathode of main discharge is presented on Figure 4. 


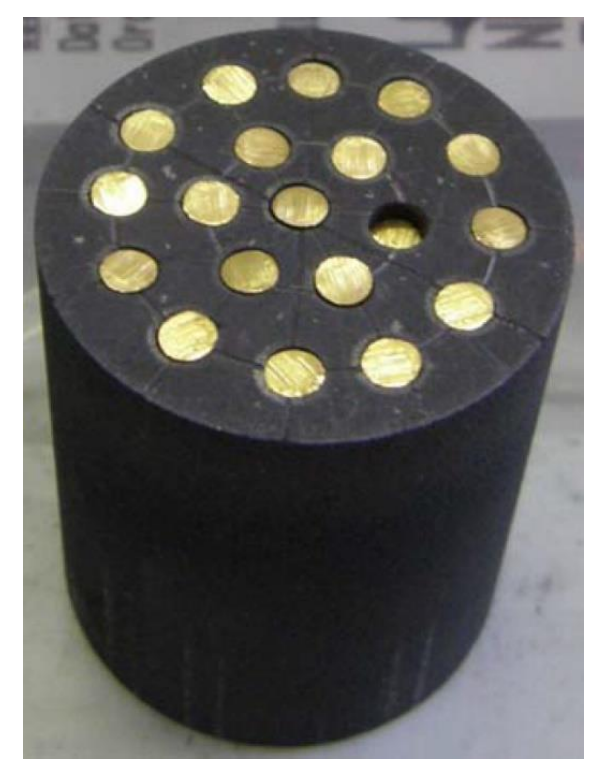

Figure 4. The example of design of a cylindrical cathode of main discharge, containing cylindrical rods from different materials, for instance, silver, tungsten, gold etc. This is item $1 \mathrm{n}$ Figure 3, [11].

The capacitors C1 and C2 are connected to a power supply unit with varied voltage (100-300 V). The ignition electrodes are supplied by the pulsed voltage with an amplitude of $800 \mathrm{~V}$. The inductance $\mathrm{L}$ are used for reduce the current growth rate to a required value. The growth of the carbon nano-matrix is enhanced by irradiation with Ar ions. The flux of Ar ions is formed by a low-pressure ion source, installed in a separate section of the deposition reactor chamber, (Fig. 2).

The energy of ions bombarding the substrate surface depends on the substrate bias voltage, being varied in the range $0-300 \mathrm{eV}$ by both the carbon plasma parameters and the ion source extractor voltage, depending on the parameters of the plasma assisted deposition. The nano-matrix can be deposited onto Si wafer, metals, and $\mathrm{NaCl}$ single crystals at an ion energy of $150 \mathrm{eV}$. Before deposition, the reactor chamber was pumped down to the residual pressure of $10^{-4} \mathrm{~Pa}$. The operating pressure during the deposition is $10^{-4} \mathrm{~Pa}$. The structure of bonds in the grown carbon nano-matrixes can be programmed by the processes of selforganization and auto-synchronization of the growing nanostructures.

Existence of electrical conductivity anisotropy of the two-dimensionally ordered linear-chain carbon nanomatrix opens possibility for control of the nano-additives spatial orientation and properties in the reaction zone through the external electromagnetic fields. In this connection, we can use additional possibility for control by heat transfer into the EM through application of the manipulated electrostatic field and, hence, control the EM regression rate. 


\section{The Electrostatic Field Effects}

The electrostatic field powers up the reaction rate and can deform the combustion front, change the mechanism of phase transformations, and change the flame temperature. The electrostatic field can act both as a catalyst and a combustion inhibitor, depending on its configuration and location, [12-14].

For demonstration the fundamental principle of the electrostatic field influence on the combustion process, that we propose to use in conjunction with programming the spatial structure and physics-chemical properties of the functionalized carbon-based nano-additives, we will consider a specific example for the hybrid propulsion system, [12-14]. The electrostatic field generation system for study of influence of the electrostatic field on the combustion process in the hybrid propulsion system is presented in Figure 5, [1214].

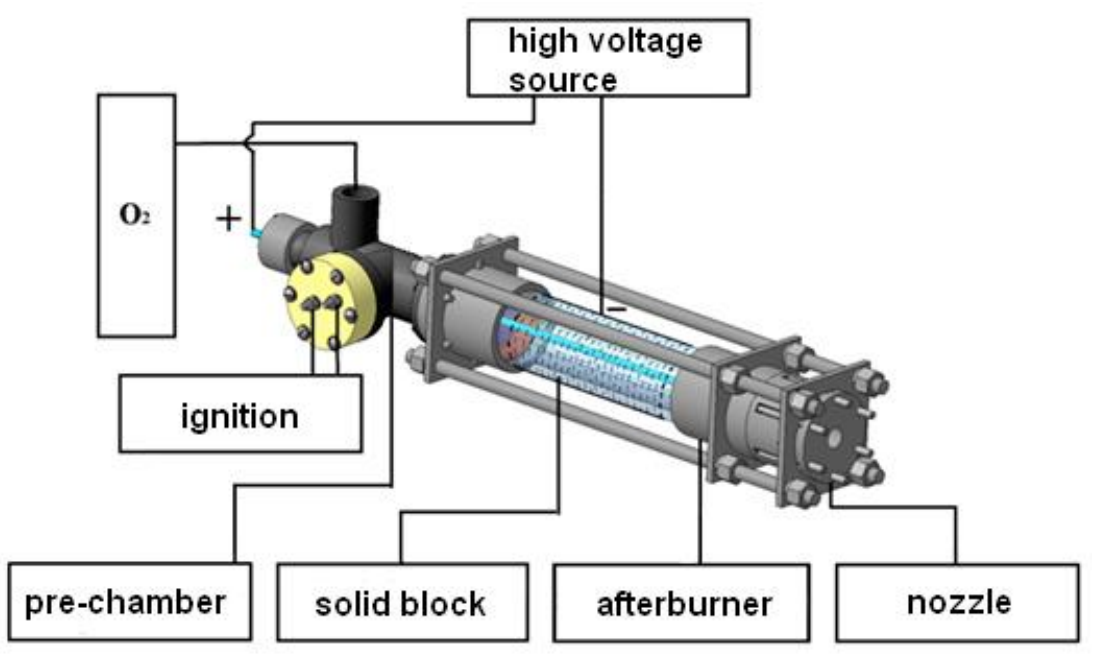

Figure 5. The electrostatic field generation system for study of influence of the electrostatic field on the combustion process in the hybrid propulsion system, [12-14].

The electrostatic field is created between two electrodes: the first electrode is located along the engine axis, and the second - outside of the fuel grain. The configuration of the used electrodes provides conditions in which the charged particles flow near to the burning surface thus increasing the heat flow into the burning surface and, as a consequence, the burning rate. Investigation of the effect of the electrostatic field on the thrust in the hybrid rocket motor for the fuel vapor polymethylmethacrylate (PMMA) - gaseous oxygen is presented on Figure 6, [12-14]. 


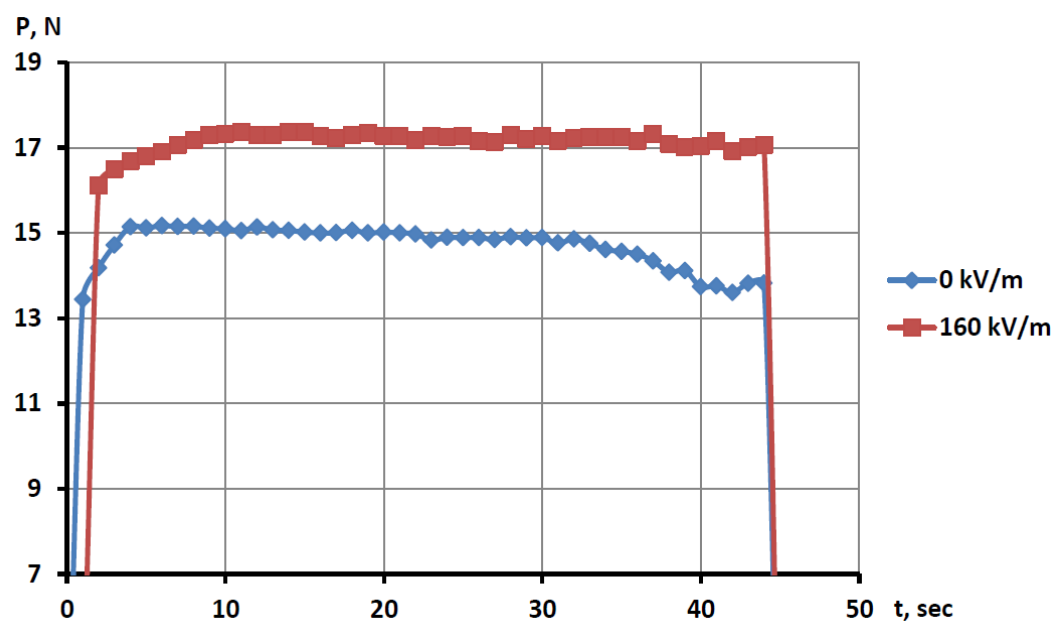

Figure 6. Time dependence of thrust at an oxidizer flux density of $23 \mathrm{~kg} / \mathrm{m}^{2} \mathrm{~s}$ (for polymethylmethacrylate (PMMA) grain), [12-14].

Investigation of the effect of an electrostatic field on the thrust in a hybrid rocket motor for a polyamide 6 oxygen gas fuel pair is presented on Figure 7, [12-14].

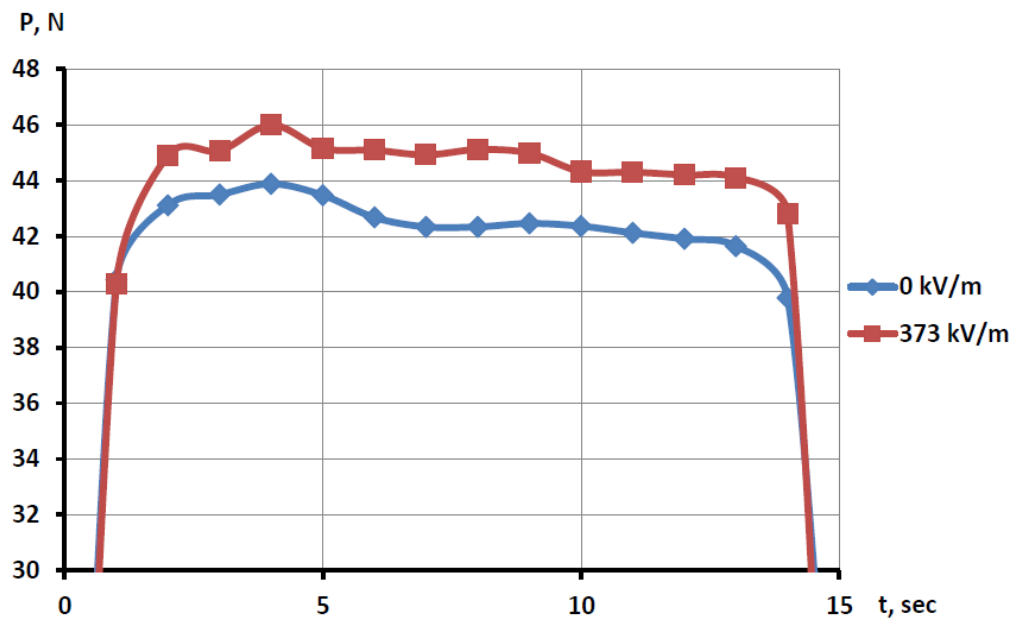

Figure 7. Time dependence of thrust at an oxidizer flux density of $61 \mathrm{~kg} / \mathrm{m}^{2} \mathrm{~s}$ (polyamide 6), [12-14].

We can observe, that the engine thrust increases in all ranges of oxidant flow rates in the presence of an electrostatic field. On the Figure 8 presented example of the electrostatic field influence on the selforganized wave patterns formation on the surface of polyamide-based fuel grain in hybrid rocket motor. 


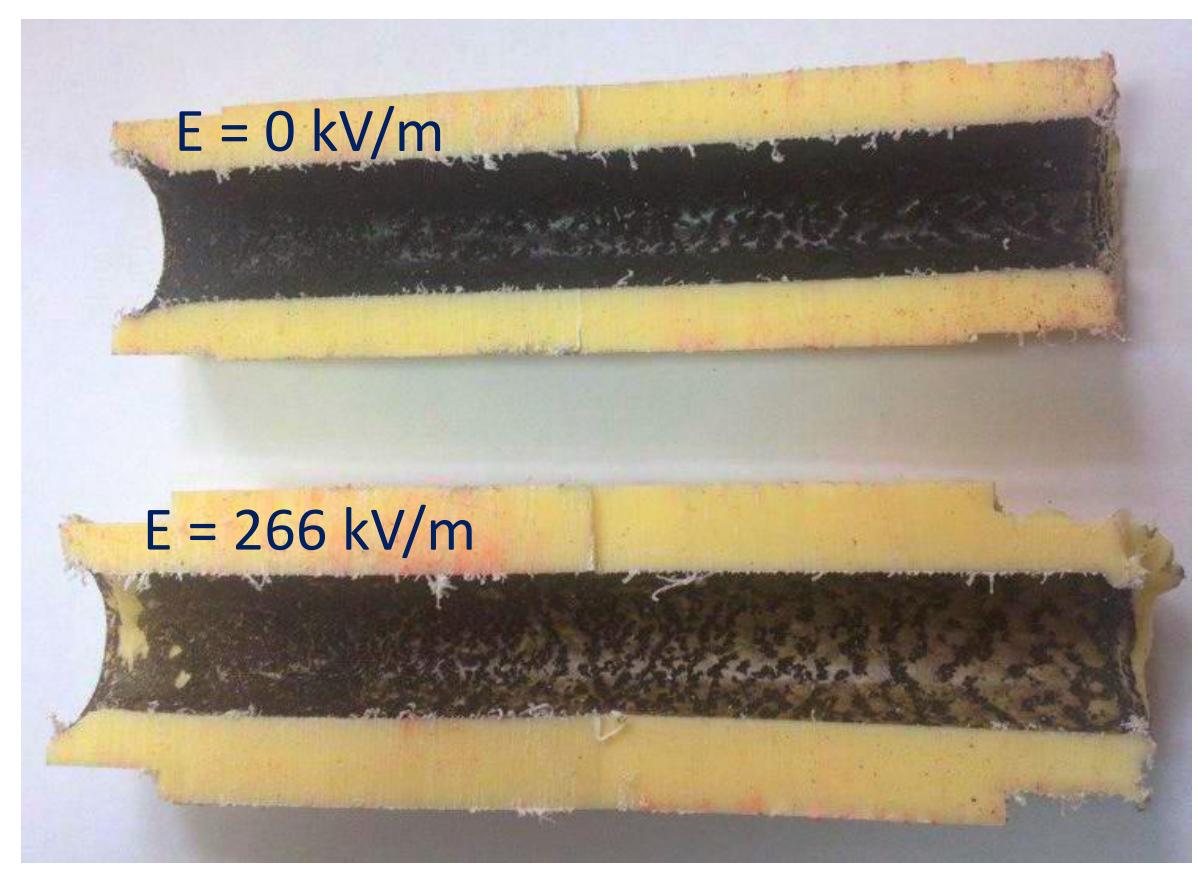

Figure 8. Self-organized wave patterns observed on the surface of polyamide-based fuel grain in hybrid rocket motor at the oxidizer $\left(\mathrm{O}_{2}\right)$ mass flux rate $61 \mathrm{~kg} / \mathrm{m}^{2} \mathrm{sec}$ (a polyamide 6 plastic fuel) without and with influence of the electrostatic field, [12-14].

\section{Synergistic Effect in the Energetic Material Reaction Zones}

The micro- and nano- scale structures of the EM reaction zones can be classified as the synergetic objects. In the EM reaction zones exists necessary conditions for realization of the phenomenon of selfsynchronization. First of all this is a set of similar micro- and nano- scale oscillatory structures, which have an identical information-algorithmic condition and being in the conditions supposing fast information exchange between them. Fluctuating micro- and nano- scale structures also are generators of the acoustic waves and electro-magnetic radiation. Synchronization of a large number of oscillators is a well-known form of collective behavior. These phenomena are universal. Oscillators with similar frequencies can obviously synchronize when phase-minimizing coupling acts between them.

At joint using of the functionalized carbon-based nano-additives with modified structure under the influence of electrostatic field, we can observe the synergistic effects in the EM reaction zones, that can be used for enhancement of the EM performances. Simultaneous interaction of these two factors in the reactionary zones will intensify self-synchronization phenomenon.

The two-dimensionally ordered linear chain carbon nano-matrix also can be considered as both acoustic and electromagnetic sensitive nanostructured metamaterial. Carbon-based nano-additives in the EM 
reaction zones can be oriented and distributed along the lines of force of the electric field.

The new fundamental possibilities for predictive programming both by the spatial structure and physicschemical properties of the functionalized carbon-based nano-additives is connected with application of the data-driven carbon nanostructures genome approach. In accordance with the data-driven carbon nanostructures genome approach, for every specific carbon nanostructured metamaterial, there is a specific set of universal relationships like as: "Growth Regimes - Structure - Properties", that allows one to unambiguously predict the main characteristics of the nanostructures under design and the technological regimes required for their predictive growth.

Data-driven genome approach opens new possibilities for programming of the spatially controlled growth, structural self-organizing and cluster-assembling of the two-dimensionally ordered linear chain carbon nanomatrix during the ion-assisted pulse-plasma deposition.

\section{Conclusion}

A novel concept and technologies for the EM performance enhancement by application of the new synergistic effect has proposed. Manipulating by the self-organized wave patterns excitation, by the properties of the EM reaction zones and by localization of the energy release areas can be provided through application of the functionalized carbon-based nanostructured metamaterials as a nano-additives along with simultaneous manipulating by their properties through the electrostatic field. At joint using of the functionalized carbon-based nano-additives with programmable structure under the influence of electrostatic field, we can observe the synergistic effects, that can be used for enhancement of the EM performances. Suggested concept for manipulating by self-organized wave patterns and by oscillatory networks of the micro- and nano-scale structures in the EM reaction zones is opening the door for completely new ways for producing extremely small thrust impulses for the extra-precise attitude control for the deep-space-capable small satellites.

\section{Acknowledgments}

The research work is jointly supported and funded by the Scientific and Technological Research Council of Turkey (TÜBITAK) and the Russian Foundation for Basic Research (RFBR) according to the research project № 20-58-46014. 


\section{References}

[1] Jenny, H., Cymatics: A Study of Wave Phenomena \& Vibration, 3rd ed. Macromedia Publishing, New Hampshire, USA, 2001.

[2]. Yan, Q.-L., Zhao, F.-Q., Kuo, K.K., Zhang, X.H., Zeman, S., DeLuca, L.T., Catalytic Effects of Nano Additives on Decomposition and Combustion of RDX-, HMX-, and AP-Based Energetic Compositions, Progress in Energy and Combustion Science, Vol. 57, 2016, pp. 75-136, https://doi.org/10.1016/j.pecs.2016.08.002.

[3]. Yan, Q.-L., Gozin, M., Zhao, F.-Q., Cohen, A., Pang, S.-P., Highly Energetic Compositions Based on Functionalized Carbon Nanomaterials, (Review Article) Nanoscale, 2016, 8, 4799-4851, DOI: 10.1039/C5NR07855E

[4]. Elanjickal, S., Gany, A., Enhancement of the Fuel Regression Rate in Hybrid Propulsion by Expandable Graphite Additive, Combustion Science and Technology, 2020, 192:7, 1253-1273, https://doi.Org/10.1080/00102202.2020.1748017

[5]. Muller, G.T., Gany, A., Burning Phenomena of a Polymeric Fuel Containing Expandable Graphite, Propellants, Explosives, Pyrotechnics, Vol. 45, Issue11, 2020, pp. 1764-1768, https://doi.org/10.1002/prep.202000138

[6]. Shevchenko, N.V., Gorbachev, V.A., Ubey-Volk E.Yu. et al, The Influence of Detonation Nano-Diamonds on the High Energy Rocket Propellants Combustion Characteristics, Composite Materials Constructions, № 3 (135), 2014, pp. 33-39, (in Russian).

[7]. Gorbachev, V.A., Ubey-Volk E.Yu., Shevchenko, N.V., Golubev, A.A., Detonation Nano-Diamonds as a Prospective Component of the Composite Solid Rocket Propellants, Russian Journal of Chemistry and Chemical Technology, 2016. Vol. 59, № 8, pp. 96-100, (in Russian).

[8]. Zhang, W., Xiong, H., Wang, S., Li, M., Gu, Y., Electromagnetic Characteristics of Carbon Nanotube Film Materials, Chinese Journal of Aeronautics, Vol. 28, Issue 4, 2015, pp. 1245-1254, https://doi.org/10.1016/j.cja.2015.05.002. 
[9]. Shi, L., Rohringer, P., Suenaga, K. et al. Confined Linear Carbon Chains as a Route to Bulk Carbyne, Nature Mater 15, 634-639 (2016). https://doi.org/10.1038/nmat4617

[10]. Flood, P., Babaev, V., Khvostov, V., Novikov, N., Guseva, M., Carbon Material with a Highly Ordered Linear-Chain Structure, In Polyynes Synthesis, Properties, and Applications; Cataldo, F., Ed.; Taylor \& Francis Group: Didcot, UK, 2005, pp. 219-252. https://doi.org/10.1201/9781420027587

[11]. Arc-Pulse Carbon Plasma Source Technologies, SWISSIMPIANTI Sagl, Switzerland, https://www.swissimpianti.ch/technology

[12]. Reshetnikov, S.M., Zyryanov, I.A., Budin, A.G., Reshetnikov, I.S, Hybrid Rocket Engine Control by the Electrostatic Field, Engineering Science and Technology Review, 11 (1) (2018), pp. 146 - 150, doi:10.25103/jestr.111.17

[13]. Reshetnikov, S.M., Zyryanov, I.A., Budin, A.G., Pozolotin, A.P., Method of Increase the Hybrid Rocket Engine Thrust, Pat. № 2598984 of Russia, F02K 9/72, Appl. № 2015100924/06, Priority 12.01.2015, (in Russian).

[14]. Budin, A.G., Intensification of Combustion of the Polymer Block of a Hybrid Rocket Engine Through Application of the Electrostatic Field, PhD thesis, Vyatsky State University, Kirov, 2017, 165 p., (in Russian). 